\title{
Quantify the fielding performance in cricket via bayesian approach
}

\begin{abstract}
Saikia, Bhattacharjee \& Lemmer ${ }^{1}$ proposed two fielding performance measure called Preparatory Fielding Performance Measure (PFPM) and Fairer Fielding Performance Measure (FFPM) through the ball-by-ball information of a match or series of matches. The FFPM is reasonably better measure than PFPM if the purpose is to compare the fielding performances of players irrespective of their fielding positions. Therefore, FFPM can be used to rank the fielders in cricket (like ICC player ranking) based on their on-field activities. However, collecting ball-by-ball information for the series of cricket matches is a tedious job. Moreover, in the absence of any optical observation system, the entire ball-by-ball information collections, as well as computation, have to be done manually by watching the off-line video of the match. Thus, this study proposes an extension over the FFPM using Bayesian approach. The proposed fielding measure has basically two parts in which it considers ball-by-ball information of some matches of a series and scorecard information of the remaining matches. In order to demonstrate the measure, fifteen (15) cricketers are selected from the fourth season of Indian Premier League (IPL). Accordingly, the best fielder is identified among the 15 selected cricketers by ranking them on the basis of on-field activities in the fourth season of IPL.
\end{abstract}

Keywords: cricket, fielding, ipl, performance measurement, sports
Volume I Issue 4 - 2017

\author{
Hemanta Saikia,' Dibyojyoti Bhattacharjee, ${ }^{2}$ \\ Hoffie H Lemmer ${ }^{3}$ \\ 'Assistant Professor of Statistics, College of Sericulture, Assam \\ Agricultural University, India \\ ${ }^{2}$ Professor and Head, Department of Statistics, Assam University, \\ India \\ ${ }^{3}$ Emeritus Professor, Department of Statistics, University of \\ Johannesburg, South Africa
}

Correspondence: Hemanta Saikia, Assistant Professor of Statistics, Assam Agricultural University, India, Tel +919954I54094,Email h.saikia456@gmail.com

Received: August 29, 2017 | Published: September 22, 2017
Abbreviations: FFPM, fairer fielding performance measure; PFPM, preparatory fielding performance measure; IPL, Indian premier league

\section{Introduction}

Performance statistics are often used to indicate the level of achievement of the players in sports. ${ }^{2}$ Cricket is a team sport that prides itself on the fact that statistics is generated in each and every ball of the game in terms of players, teams, games and seasons. Cricket heavily relies on performance statistics to measure the performance of its players. For example - strike rate, batting average, the percentage of contribution to the team total, etc. for batsmen; and bowling average, economy rate, bowling strike rate, etc. for bowlers. ${ }^{3}$

Several performance measures which combines traditional performance statistics to measure the overall performance of batsmen and bowlers have already been proposed by different authors like Kimber \& Hansford, ${ }^{4}$ Croucher,,${ }^{5}$ Beaudin \& Swartz, ${ }^{6}$ Barr \& Kantor, Lemmer, ${ }^{8,9}$ Lewis, ${ }^{10}$ Damodaran, ${ }^{11}$ Maini \& Narayanan, ${ }^{12}$ Gerber \& Sharp, ${ }^{13}$ Suleman \& Saeed,,${ }^{14}$ Borooah \& Mangan ${ }^{15}$ and others. Saikia Bhattacharjee \& Bhattacharjee ${ }^{16}$ proposed a model that can be used to quantify the batting, bowling and wicket keeping performance of cricketers based on the data from the scorecard of a match. A performance measure for wicket keepers in cricket, that combined the dismissal rate (number of catches taken and stumpings done per match) and batting performance has been developed by Lemmer. ${ }^{17}$

However, the game of cricket is yet to have a proper measure for quantifying fielding performance. The fielding performances that are perceivable from the scorecard of a match are number of catches and run-outs. But a fielder does more than that in the field which is not expressed in the scorecard unlike the other prime skills of the game like batting and bowling. International Cricket Council (ICC) releases the ranking of teams as well as batsmen, bowlers and all-rounders periodically on the basis of on-field performance of the cricketers. But there is no ICC ranking for fielders in the game of cricket.

Fielding in cricket is the on-field action of a fielder. A fielder in a cricket match always tries to prevent the scoring of runs that the batsman desires to get by quickly returning the ball to the stumps. He also tries to get the batsman out by catching the ball or by executing a run out. In cricket, great feats of batting and bowling usually hit the headlines but it is often ignored that good fielding too can make a crucial contribution to a team's success. ${ }^{18}$ With the advent of ODI and the recent format of Twenty 20 cricket, fielding has become more athletic and is developing as a discipline to rival batting and bowling in the interest of spectators. ${ }^{19}$ Players too are becoming more and more concern about their fielding performance nowadays. They have started believing the fact that effective and tight fielding can help their team to win the match.

Traditionally, only two factors have been considered as the performance indicator of fielders in cricket - number of catches taken and run outs accomplished due to the availability of only these two factors in the scorecard of a match. However, it can be easily observed that there are several other factors involved in fielding. Even in considering catches and run outs, other factors such as the difficulty level and the accuracy of such actions need to be considered. Therefore, to provide an accurate measure for fielding performance one has to take into account all the subtle nuances involved in the process of fielding. Such an approach which considers all these factors will require ball-by-ball information of a match.

Gerber \& Sharp ${ }^{13}$ proposed a fielding performance index for the game of cricket. According to that measure, the fielding ability of a player is subject to the number of specialist fielders that are available in the team. Since fielding of a player mostly depends on his own 
skill and not on the skills of any other specialist fielder. Thus the measure has got some obvious faults and limitations. Considering the loopholes in Gerber \& Sharp ${ }^{13}$ proposed measure and the need for a measure which can analyse all the critical issues as discussed above Saikia Bhattacharjee \& Lemmer $^{1}$ proposed two fielding performance measures called the preparatory fielding performance measure (PFPM) and a refined measure called the fairer fielding performance measure (FFPM) based on ball-by-ball information of a match or a series of matches. These two measures can be used to rank the fielders in the game of cricket.

It is obvious that a fielding measure based on ball-by-ball information shall precisely look into all details of a fielder's activities in a match. However, to judge the actual quality of a fielder it is necessary to consider the performance details of a player from a considerable number of matches instead of just one or two. In the words of Lewis, ${ }^{10}$ "...the effects of outstanding or poor, single performances are smoothed over the larger number of games". But to use the measures defined by Saikia, Bhattacharjee and Lemmer ${ }^{1}$ for several matches, the job of data collection becomes tedious. The ball-by-ball information necessary for using the PFPM and FFPM is to be collected by watching the off-line video. In the absence of any optical observation system, the entire exercise is to be done manually which makes the measure really time-consuming. This makes both the measures defined in Saikia, Bhattacharjee \& Lemmer ${ }^{1}$ difficult to use beyond a fewer number of matches. Thus, this study proposes an extension over the fielding performance measures defined in Saikia, Bhattacharjee \& Lemmer. ${ }^{1}$ To demonstrate the measure, fifteen (15) cricketers are selected from the fourth season of Indian Premier League (IPL-IV) and their fielding performances are quantified.

\section{Fielding performance measure}

A reasonable fielding measure requires ball-by-ball information from a larger number of matches, but collecting the same beyond fewer matches is hectic and time-consuming. In this study, the proposed measure is a negotiation between the two. It has two parts (A and B). Part A is an improved version of the FFPM value of a said player measured from the ball-by-ball information of few matches (generally three) of a series. It is computed using the process discussed in Saikia, Bhattacharjee \& Lemmer ${ }^{1}$ and then extended with a Bayesian paradigm. Part B is computed from the scorecard information of the remaining matches of the series from which ball-by-ball information was not collected.

Thus, the proposed fielding performance measure considers ball-by-ball information of some matches of a series and scorecard information of the remaining matches. The proposed fielding performance measure can be defined as

$$
F P_{j}=A_{j}+B_{j}
$$

where $A_{j}$ is the average FFPM of the $j^{\text {th }}$ player through the ball-byball information of few matches of a series and later improved using a Bayesian model and $B_{j}$ is the fielding performance score of the $j^{\text {th }}$ player through the scorecard of the remaining matches of the series.

\section{Fielding Performance Measure based on ball-by-ball information of the match $\left(A_{j}\right)$}

In cricket, the fielding positions can basically be divided into two parts viz. close fielding which is within the 30 yards circle and distant fielding outside the 30 yards circle. Under these two fielding positions, three different aspects of fielding (i.e. catch, picking the ball and run out) can be observed as on-field performances of the cricketers. A good and active fielder always tries to catch the ball in flight or to throw it as hard and quickly as possible, irrespective of the fielding position. In addition, one good throw in a cricket match may play a vital role, as a run out can lead to the loss of a batsman of the opposing team which may even turn the outcome of the match. To consider all these aspects of fielding, ball-by-ball information of a player in a match is required. Ranges of parameters are considered and scores are assigned to these parameters based on the on-field performances of the fielders. The parameters considered for the study and their corresponding scores can be seen in Appendix-A.

Clarke $^{2}$ states that a cricketer's innings score can be modelled as

$$
S=X_{1}+X_{2}+\ldots+X_{N}
$$

Where $X_{i}$ is the score by the cricketer on the $i^{\text {th }}$ ball faced and $N$ is a random variable, representing the number of balls faced. Thus, the average batting performance of a batsman and his variation or consistency in that innings can be measured by

$$
\begin{aligned}
& E(S)=E(X) \cdot E(N) \\
& \operatorname{Var}(S)=E(X)^{2} \cdot \operatorname{Var}(N)+E(N) \cdot \operatorname{Var}(X)
\end{aligned}
$$

This concept has been thoroughly used to measure the fielding performance of the cricketers through the ball-by-ball information of a match.

Let $Y_{i j}$ 's $\left(i=1,2, \ldots, N_{j}\right)$ be the fielding performance score of the $j^{\text {th }}$ player in the $i^{\text {th }}$ ball that he fielded in a match or series of matches. The values of $Y_{i j}$ 's are obtained in the same manner as the values of $X_{i j}$ 's were obtained in Saikia, Bhattacharjee \& Lemmer. ${ }^{1}$ Then the fielding performance scores for the $j^{\text {th }}$ cricketer can be modelled as

$$
A_{j}=Y_{1 j}+Y_{2 j}+\ldots+Y_{N_{j} j}=\sum_{i=1}^{N_{j}} Y_{i j}
$$

Where $N_{j}$ is the random variable, being the number of balls fielded by the $j^{\text {th }}$ player in a given match. Hence, the average fielding performance of $j^{\text {th }}$ cricketer and his level of consistency in fielding can be measured by

$$
\begin{aligned}
& E\left(A_{j}\right)=E\left(Y_{i j}\right) \cdot E\left(N_{j}\right) \\
& \operatorname{Var}\left(A_{j}\right)=E\left(Y_{i j}\right)^{2} \cdot \operatorname{Var}\left(N_{j}\right)+E\left(N_{j}\right) \cdot \operatorname{Var}\left(Y_{i j}\right)
\end{aligned}
$$

Here the values of $\mathrm{E}\left(Y_{i j}\right)$ are estimated using a Bayes' estimate as explained below. Let $\mathrm{E}\left(Y_{i j}\right)=\theta_{i j}\left(i=1,2, \ldots, N_{j}\right)$ be the average fielding performance scores of $j^{\text {th }}$ cricketer in $i^{\text {th }}$ ball but the actual value of $\theta_{i j}$ is unknown. Let the observed average fielding performance score of $j^{\text {th }}$ cricketer in the $i^{\text {th }}$ ball called as $t_{i j}$, is calculated using the Fairer Fielding Performance Measure (FFPM) and it is given by,

$$
t_{i j}=\frac{\sum_{i=1}^{N_{j}}\left[\left(1-\beta_{i}\right) k_{i j} Y_{i j}+\beta_{i} b_{i} k_{i j} Y_{i j}\right]}{\sum_{i=1}^{N_{j}}\left[\left(1-\beta_{i}\right) k_{i j}+\beta_{i} b_{i} k_{i j}\right]}
$$


where $\quad N_{j}=$ number of balls fielded by the $j^{\text {th }}$ fielder

$Y_{i j}=$ fielding performance scores of the $j^{\text {th }}$ fielder in the $i^{\text {th }}$ ball

$k_{i j}=$ weights for the fielding activity performed by the $j^{\text {th }}$ player in the $i^{\text {th }}$ ball fielded fielder

$b_{i}=$ weights for the batsmen dismissed in the $i^{\text {th }}$ ball by $j^{\text {th }}$

and $\beta_{i}$ is an indicator variable where

$\beta_{i}=\left\{\begin{array}{l}1, \quad f \text { the fielding activity } \boldsymbol{a} \text { the } i^{h} \text { ball leads } b \text { a dissmissal } \\ 0, \quad \text { otherwise }\end{array}\right.$

The process of obtaining the values of $Y_{i j}$ and $k_{i j}$ is same as that of $X_{i j}$ and $w_{i j}$ respectively in Saikia, Bhattacharjee \& Lemmer. ${ }^{1}$

The values of $t_{i j}$ in (8) are difficult to attain as it is dependent on $Y_{i j}$ To obtain the values of $Y_{i j}$, one has to record ball by ball information of the matches. As discussed earlier, in the absence of any software/ optical observation system for converting fielding performance of players to the values of $Y_{i j}$ the researcher has to obtain them by watching the off-line video. This may be difficult to perform in large scale i.e. for a larger number of matches. This makes the observed measure of quantification of fielding performance i.e. $Y_{i j}$ vis-à-vis $t_{i j}$ weak in the sense that it is not based on many matches. Thus, the average fielding performance scores of $j^{\text {th }}$ cricketer in $i^{\text {th }}$ ball i.e. $\theta_{i j}$ cannot be estimated from the corresponding observed measure $t$ without proper refinement. Based on the available values of $t_{i j}$ and considering $\theta_{i j}$ to be a stochastic variable, a Bayesian model can be developed which can provide refined estimates for the average fielding performance score of a cricketer. This provides the motivation for the quantification of fielding performance in a Bayesian paradigm. Thus, $\theta_{i j}$ be a random variable and let $E\left(\theta_{i j}\right)=\mu_{i j}$ and $\operatorname{Var}\left(\theta_{i j}\right)=\sigma_{i j}^{2}$. Under this assumption, it can be demonstrated that the best Bayes' estimate of $\theta_{i j}$ is a linear combination of the observed rate $t_{i j}$ and $\mu_{i j}{ }^{20}$ The estimated value of $\theta_{i j}$ is -

$$
\theta_{i j}=w_{j} t_{i j}+\left(1-w_{j}\right) \mu_{i j} \text { where } 0 \leq w_{j} \leq 1
$$

This estimator is useful in improving the estimation by reducing the mean squared error towards zero. In equation (9), one part of the Bayes' estimate of $\theta_{i j}$ is determined from the observed fielding performance score of the $j^{\text {th }}$ player in $i^{\text {th }}$ ball and hence is deterministic for a given sample (i.e. $t_{i j}$ ). However, the other part of this estimate is stochastic $\left(i . e . \mu_{i j}\right)$. So one can generate $95 \%$ credible interval also for the stochastic part and hence could express the estimated value of $\theta_{i j}$ in terms of an interval instead of a point value. The interval estimate of a parameter is a robust approach compared to the point estimate, where one can express with a given level of confidence the expected range in which a given parameter is supposed to lie (Freund, 1992). The value of $w_{j}$ is given by -

$$
w_{j}=\frac{\sigma_{i j}^{2}}{\sigma_{i j}^{2}+\frac{\mu_{i j}}{N_{j}}}
$$

The Bayes' estimate of $\theta_{i j}$ is given in (9) is also called the shrinkage estimator. This estimate shrinks or moves the individual estimate $t_{i j}$ towards the pooled estimate $\mu_{i j .}{ }^{21}$ However there is no reason to consider that $\mu_{i j}$ and $\sigma_{i j}^{2}$ are same for all the players and thus the bootstrap technique is used to determine the values of $\mu_{i j}$ and $\sigma_{i j}^{2}$ for each player independently.

\section{Estimating the values of $\mu_{i j}$ and $\sigma_{i j}^{2}$ using bootstrap technique}

Bootstrap technique is a powerful tool for constructing inferential procedures in modern statistical data analysis. It is an alternative to the traditional statistical technique of assuming a particular probability distribution. The usual assumption about data that are being bootstrapped is that the observations are independent and identically distributed..$^{22}$ It is a method for estimating the distribution of an estimator or test statistics by re-sampling the data. ${ }^{23}$ The data are generated by sampling the available data randomly with replacement. For example - from a sample, suppose we have got one statistics (assume mean) but we do not know the confidence interval of this mean or distribution of this mean. In such cases, the bootstrap technique gives more detail information on the distribution or confidence interval of this mean.

Based on the ball-by-ball fielding scores of the $j^{\text {th }}$ player for $i^{t h}$ ball that he fielded, the distributional pattern (i.e. relative frequency) of the observed fielding score is arranged. As computation based on large samples will help to obtain consistent estimates of the parameters ${ }^{24}$ the bootstrap technique is used to generate 100 such samples of size 1000 each. The distribution of sample means is obtained using the Central Limit Theorem (CLT) which in turn can be gainfully applied to estimate the values of $\mu_{i j}$ and $\sigma_{\ddot{j}}^{2}$. The CLT states that for any population distribution with mean $\mu$ and standard deviation $\sigma$, the sampling distribution of the sample mean $\bar{X}$ is approximately normal with mean $\mu$ and standard deviation $\sigma / \sqrt{ } n \cdot{ }^{25}$ Symbolically, it can be expressed as

$$
f(\bar{x} \mid \mu, \sigma, n) \sim N\left(\mu, \frac{\sigma}{\sqrt{n}}\right)
$$

Thus, the values of $\mu_{i j}$ and obtained in this way can be replaced in (10) and (9) and then in (6) to quantify the average fielding performance of that player in IPL-IV. This fielding performance measure $\left(A_{j}\right)$ is demonstrated for 15 selected cricketers using the data from a few Twenty 20 cricket matches in the fourth season of the IPL. Based on the parameters considered in Appendix-A, the relevant ballby-ball information for the selected cricketers is collected through observing the off-line video of the matches. The video of these matches is downloaded from the website www.youtube.com. Detailed information of the matches that are considered here is provided in Table 2.

Bootstrap technique (as mentioned) is used to generalize the fielding performance of selected cricketers from the fourth season of the IPL. Accordingly, the fielding performances of the 15 cricketers are quantified by $\mathrm{E}\left(A_{i}\right)$ and can be seen in column 3 of Table 1 .

\section{Fielding performance measure through scorecard of the match $\left(B_{j}\right)$}

Let $X_{i j}$ be the performance score of the $j^{\text {th }}$ player for the $i^{\text {th }}$ factor ( $i=1$ represents catch and $i=2$ represents run-outs) obtained from the 
scorecard of the remaining matches of the series. Suppose the $j^{\text {th }}$ fielder has taken $n_{1}$ catches and is involved in $n_{2}$ run-outs. Then, $X_{i j}$ is defined as the sum of the wicket weights of those batsmen whose wickets have been lost due to the $j^{\text {th }}$ player through factor $i$ ( $=1$ represents catch and $=2$ represents run-outs). Thus performance score of the $j^{\text {th }}$ fielder,

$$
X_{i j}=\sum_{k=1}^{n_{i}} b_{i j k} \text { for } i=1,2
$$

Where $b_{i j k}=$ weights of the $k^{\text {th }}$ batsman dismissed by the $j^{\text {th }}$ player through the $i^{\text {th }}$ factor

A natural call for $X_{i j}$ would have been adding up the number of dismissals under the $i^{\text {th }}$ factor. But when a fielding activity leads to the loss of the wicket of a top order batsman it shall have a higher weight compared to a batsman lower down the order. By making use of the wicket weights for ODIs developed by Lemmer ${ }^{9}$ the weighting factor $b_{j}$ is also incorporated into the formula - see Appendix-B. The weights depend on the batting position of the batsmen whose wicket has been taken.

Now both the factors catch and run out are in positive dimension i.e. more the number of catch and run out executed by a player better is his fielding skill. Then it is normalized as

$$
Z_{i j}=\frac{X_{i j}-\min \left(X_{i j}\right)}{\max _{j}\left(X_{i j}\right)-\min _{j}\left(X_{i j}\right)} \text { for } i=1 \text { (catch), } 2 \text { (run-out) }
$$

\begin{tabular}{|c|c|c|c|c|c|}
\hline \multirow[t]{2}{*}{ Players name } & \multirow[t]{2}{*}{$\begin{array}{l}\text { No. of balls } \\
\text { fielded }\end{array}$} & \multirow[t]{2}{*}{$\begin{array}{l}\text { Estimated average fielding } \\
\text { performance } E(\mathbf{A j})\end{array}$} & \multirow[t]{2}{*}{$\begin{array}{l}\text { Variability in fielding } \\
\text { performance } \operatorname{Var}(A j)\end{array}$} & \multicolumn{2}{|c|}{$\begin{array}{l}\text { Credible interval } \\
(95 \%)\end{array}$} \\
\hline & & & & Upper & Lower \\
\hline RP Singh & 22 & 11.132 & 2.038 & 11.154 & 11.11 \\
\hline MS Dhoni & 57 & 26.619 & 3.445 & 26.676 & 26.562 \\
\hline V Kohli & 34 & 18.734 & 4.247 & 18.768 & 18.666 \\
\hline Y Singh & 27 & 15.174 & 2.168 & 15.201 & 15.12 \\
\hline R Sharma & 22 & 11.418 & 2.525 & 11.462 & 11.396 \\
\hline Y Pathan & 33 & 14.916 & 1.985 & 14.949 & 14.883 \\
\hline Z Khan & 21 & 11.193 & 1.672 & 11.214 & 11.172 \\
\hline I Pathan & 28 & 16.324 & 2.61 & 16.352 & 16.296 \\
\hline JH Kallis & 22 & 12.034 & 2.588 & 12.078 & 12.012 \\
\hline A Reddy & 18 & 9.378 & 1.078 & 9.414 & 9.36 \\
\hline K Pollard & 27 & 13.824 & 2.362 & $|3.85|$ & 13.743 \\
\hline DJ Bravo & 21 & 13.356 & 1.456 & 13.377 & 13.335 \\
\hline JA Morkel & 23 & 14.72 & 3.001 & 14.743 & 14.697 \\
\hline M Hayden & 15 & 6.855 & 0.746 & 6.87 & 6.84 \\
\hline S Dhawan & 19 & 10.849 & 2.265 & 10.868 & 10.811 \\
\hline
\end{tabular}

Table I Fielding performance of I5 selected cricketers in IPL-IV quantified by A

This process of normalization limit the performance indicator values within an interval of 0 and 1 which is always non-negative and free of the unit. Now considering catch and run out as performance indicators for fielding skill of a cricketer, the fielding performance measure of $j^{\text {th }}$ player is denoted by

$$
B_{j}=\sum_{i=1}^{2} \omega_{i} Z_{i j}
$$

$$
\begin{aligned}
& \text { Where } i=1 \text { (catch), } 2 \text { (run-out) } \\
& \text { Where } \omega_{i}=\text { weight of the } i^{\text {th }} \text { factor } \\
& \qquad Z_{i j}=\text { normalized score for the } i^{\text {th }} \text { factor of the } j^{\text {th }} \text { player }
\end{aligned}
$$

Iyenger $\&$ Sudarshan ${ }^{26}$ assumed that the weights $\left(\omega_{i}\right)$ vary inversely as the variation in the respective variables. This conception has been thoroughly applied in this study to determine the weights for catch and run-out of fielders in cricket.

If $\omega_{i}$ represent the weight of the $i^{\text {th }}$ factor then it is calculated as,

$$
\omega_{i}=\frac{C}{\sqrt{\operatorname{Var}\left(Z_{i j}\right)}} i=1,2
$$

Where $\sum^{2} \omega_{i}=1$ and $C$ is a normalizing constant for fielding skill that follows

$$
C=\left[\sum_{i=1}^{2} \frac{1}{\sqrt{\operatorname{Var}\left(Z_{i j}\right)}}\right]^{-1}
$$


The choice of the weights in this manner would ensure that the large variation in any one of the factor would not unduly dominate the contribution of the rest of the factors. ${ }^{26}$

In order to demonstrate this fielding performance measure $\left(B_{j}\right)$ the same fifteen cricketers are considered. Data from the scorecard of the remaining matches (excluding the matches mentioned in Table

Table 2 Matches of IPL-IV that were used for ball-by-ball fielding details

2) of the fourth season of the IPL, in which the players figured are considered. The desired fielding performance scores of cricketers in IPL-IV are calculated using equation (14). Based on equation (15), the weights of the catch and run-out are calculated and were found to be 0.496 and 0.504 respectively. The values of the fielding performance measure $\left(B_{i}\right)$ for 15 cricketers based on the scorecard information can be seen in Table 3 .

\begin{tabular}{llllll}
\hline SI. no. & Match no. & Home team & Away team & Date & Venue \\
\hline I & 7 & Rajasthan Royals & Delhi Daredevils & 12.4 .201 I & Jaipur \\
2 & 24 & Kolkata Knight Riders & Royal Challengers Bangalore & $22.4 .201 \mathrm{I}$ & Kolkata \\
3 & 25 & Mumbai Indians & Chennai Super Kings & $22.4 .201 \mathrm{I}$ & Mumbai \\
4 & 42 & Deccan Chargers & Kolkata Knight Riders & $3.5 .201 \mathrm{I}$ & Hyderabad \\
5 & 55 & Rajasthan Royals & Royal Challengers Bangalore & II.5.20II & Jaipur \\
6 & 56 & Chennai Super Kings & Delhi Daredevils & $12.5 .201 \mathrm{I}$ & Chennai \\
\hline
\end{tabular}

Table 3 Fielding performance measure $\left(B_{j}\right)$ obtained from scorecard of the remaining IPL-IV matches of the selected cricketers

\begin{tabular}{|c|c|c|c|c|}
\hline Player's name & $\begin{array}{l}\text { Matches played in } \\
\text { IPL-IV }\end{array}$ & $\begin{array}{l}\text { Matches used for scorecard } \\
\text { information }\end{array}$ & $\begin{array}{l}\text { Fielding performance } \\
\text { measure }(B j)\end{array}$ & Rank \\
\hline K Pollard & 14 & 12 & 0.690681 & 2 \\
\hline MS Dhoni & 13 & II & 0.779687 & I \\
\hline R Sharma & 15 & 12 & 0.567746 & 3 \\
\hline $\mathrm{JH}$ Kallis & 16 & 14 & 0.529632 & 5 \\
\hline Y Pathan & 14 & 12 & 0.567746 & 4 \\
\hline RP Singh & 12 & 10 & 0.508482 & 6 \\
\hline JA Morkel & 14 & 12 & $0.25424 I$ & 7 \\
\hline IPathan & 14 & 12 & 0.220313 & 8 \\
\hline V Kohli & 16 & 14 & 0.182199 & II \\
\hline Z Khan & 14 & 12 & 0.220313 & 9 \\
\hline DJ Bravo & 10 & 8 & 0.220313 & 10 \\
\hline Y Singh & 14 & 12 & 0.110156 & 14 \\
\hline M Hayden & 16 & 14 & 0.127121 & 13 \\
\hline S Dhawan & 10 & 9 & 0.182199 & 12 \\
\hline A Reddy & 5 & 4 & 0 & 15 \\
\hline
\end{tabular}

Table 3 reveals that MS Dhoni is the best fielder out of fifteen selected cricketers in IPL-IV with a score of 0.779 followed by K Pollard with 0.690 . However, a wicket keeper is in an advantageous position, as he gets involved in several dismissals. A Reddy is the least scorer in fielding performance and gets the last rank. However, this does not necessarily mean that A Reddy is the worst fielder of all. The fielding performance measure $\left(B_{j}\right)$, defined in equation (14) is based on the number of catches and run outs in which the player was instrumental. In IPL-IV, A Reddy neither took any catches nor executed any run-out. Moreover, he played only 5 matches. Therefore, his fielding performance score value is zero. This is a huge limitation of using only $B_{j}$ for the quantification of fielding performance. Thus, it is necessary to combine $B_{j}$ with $A_{j}$ to comprehend a players' precise and accurate fielding performance.

\section{Results and discussion}

Now by substituting the fielding performance scores of cricketers from $B_{j}$ and $A_{j}$ in equation (1), one can get the ultimate fielding performance score $\left(F P_{j}\right)$ of 15 cricketers for IPL-IV ( $c f$. T able 4). Table 4 reveals that the value of the fielding performance of MS Dhoni is 27.399. It indicates that MS Dhoni was the most active fielder out of 15 selected cricketers in the fourth season of the IPL. However, Dhoni 
might have an advantage over the others, being a wicket keeper in the team. A wicket keeper has to collect most of the balls (some of them in the form of catches) and the throws that are coming from different fielders during a match. Also, a wicket keeper has more chances of getting involved in dismissals by taking catches when the batsman edges a ball of by materializing a stumping. If we exclude MS Dhoni,
V Kohli appears to be the most dynamic fielder in IPL-IV with the value of his fielding performance 18.916 followed by I Pathan with a corresponding fielding performance score 16.544. Furthermore, based on the values of credible interval column in Table 4, one can get the expected level of fielding performance for the fifteen selected cricketers.

Table 4 Fielding performance score of 15 cricketers in IPL IV

\begin{tabular}{|c|c|c|c|c|c|c|}
\hline \multirow[t]{2}{*}{ Players Name } & \multirow[t]{2}{*}{ Aj (Col. 3 of table I) } & \multirow[t]{2}{*}{$\mathrm{Bj}$ (Col. 4 of table 3) } & \multirow[t]{2}{*}{ Fpj } & \multicolumn{2}{|c|}{ Credible interval } & \multirow[t]{2}{*}{ Rank of the fielders } \\
\hline & & & & Upper & Lower & \\
\hline K Pollard & 13.824 & 0.691 & 14.515 & 14.542 & 14.434 & 7 \\
\hline MS Dhoni & 26.619 & 0.78 & 27.399 & 27.456 & 27.342 & I \\
\hline R Sharma & 11.418 & 0.568 & 11.986 & 12.03 & 11.964 & 10 \\
\hline JH Kallis & 12.034 & 0.53 & 12.564 & 12.608 & 12.542 & 9 \\
\hline Y Pathan & 14.916 & 0.568 & 15.484 & 15.517 & $15.45 \mid$ & 4 \\
\hline RP Singh & 11.132 & 0.508 & 11.64 & 11.662 & 11.618 & 11 \\
\hline JA Morkel & 14.72 & 0.254 & 14.974 & 14.997 & 14.951 & 6 \\
\hline IPathan & 16.324 & 0.22 & 16.544 & 16.572 & 16.516 & 3 \\
\hline V Kohli & 18.734 & 0.182 & 18.916 & 18.95 & 18.848 & 2 \\
\hline Z Khan & 11.193 & 0.22 & 11.413 & 11.434 & 11.392 & 12 \\
\hline DJ Bravo & 13.356 & 0.22 & 13.576 & 13.597 & 13.555 & 8 \\
\hline Y Singh & 15.174 & 0.11 & 15.284 & 15.311 & 15.23 & 5 \\
\hline M Hayden & 6.855 & 0.127 & 6.982 & 6.997 & 6.967 & 15 \\
\hline S Dhawan & 10.849 & 0.182 & 11.031 & 11.05 & 10.993 & 13 \\
\hline A Reddy & 9.378 & 0 & 9.378 & 9.414 & 9.36 & 14 \\
\hline
\end{tabular}

\section{Conclusion}

With the increasing popularity of cricket, issues related to the quantitative analysis of cricket data have gained momentum. Statisticians and data analysts are using such data for predicting match outcome, selecting the optimized balanced team, determining the value of players and so on. Most of these analyses are based on precise quantification of players' performance. While most of the measures of quantifying players' performance take into consideration batting and bowling skills, fielding skill is often left ignored and marginalized. With increasing importance of limited over game, especially the Tewnty20 format of cricket, saving of runs has become equally important as scoring them. Thus, the necessity of including the fielding ability of a player in the evaluation performance measurement seems essential. But in the absence of any appropriate tool to quantifying fielding measurement, this has not been probably attempted.

Saikia, Bhattacharjee \& Lemmer ${ }^{1}$ developed a fielding performance measure based on the ball-by-ball information of the match. Collecting ball-by-ball information is a tedious job. In the absence of any software or optical observation system, a researcher has to watch matches ball-by-ball and perform the scoring. Thus, given the time constraint and the type of activity involved it is unlikely that information from a large number of matches can be included. Thus, this paper improvises the aforesaid measure. The current measure uses bootstrapping method to refine fielding performance scores obtained from ball-by-ball information of a few matches of the series. The index is further improvised by including the fielding performance of cricketers, through run-outs and catches (obtained from the scorecard of the other matches of the series) using a composite index.

The measure $F P_{j}$ defined in the paper can be used to quantify the fielding performances of cricketers for a series of matches, whether it is Test, ODI or Twenty 20 cricket. Individual fielding performance scores can then be aggregated to obtain the overall fielding performance of a team. Such an attempt can depict the best fielding team amongst the teams that participated in a tournament. However, benefits of this fielding measure can be realized by coaches, players and team managements only when user-friendly software is developed that can perform all necessary calculations, once appropriate data is inserted. ${ }^{27,28}$

\section{Acknowledgements}

None.

\section{Conflict of interest}

Author declares there is no conflict of interest in publishing the article 


\section{References}

1. Saikia H, Bhattacharjee D, Lemmer HH. A Double Weighted Tool to Measure the Feilding Performance in Cricket. International Journal of Sports Science and Coaching. 2012;7(4):699-713.

2. Clarke SR. Studying Variability in Statistics via Performance Measures in Sport. International Statistical Institute. 2007;56.

3. Saikia H, Bhattacharjee D, Bhattacharjee A. Performance Based Market Valuation of Cricketers in IPL. Sports, Business and Management: An International Journal. 2013;3(2):127-146.

4. Kimber AC, Hansford AR. A Statistical Analysis of Batting in Cricket. Journal of the Royal Statistical Society. 1993;156(3):443-455.

5. Croucher JS. Players Ratings in One-day Cricket. Proceedings of the Fifth Ausralian Conference on Mathematics and Computers in Sport. University of Technology, Australia; 2000. p. 95-106.

6. Beaudoin D, Swartz T. The Best Batsmen and Bowlers in One-day Cricket. South African Statist J. 2003;37:203-222.

7. Barr GDI, Kantor BS. A criterion for comparing and selecting batsmen in limited overs cricket. Journal of the Operational Reasearch Society. 2004;55(12):1266-1274.

8. Lemmer $\mathrm{H}$. The combined bowling rate as a measure of bowling performance in cricket. South African Journal of Reasearch in Sport $2002 ; 24(2): 37-44$

9. Lemmer H. A measure for the batting performance of cricket players. South African for Research in Sport, Physical Education and Recreation. 2004;26(1):55-64

10. Lewis AJ. Towards fairer measures of player performance in one-day cricket. Journal of the operational research society. 2005;56(7):804-815.

11. Damodaran U. Stochastic dominance and analysis of ODI batting performance: The Indian cricket team, 1989-2005. J Sports Sci Med. 2006;5(4):503-508.

12. Maini S, Narayanan S. The Flaw in Batting Averages. The Actuary, London; 2007. p. 30-31.

13. Garber H, Sharp GD. Selecting a Limited Overs Cricket Squad Using an Integer Programming Model. South African Journal for Research in Sport, Physical Education and Recreation. 2006;28(2):81-90.
14. Suleman MT, Saeed MA. Option on human performance: a case study of Indian Premier League. SSRN, Netherlands; 2009. p. 28.

15. Borooah VK, Mangan JE. The "Bradman Class": An Exploration of Some Issues in the Evaluation of Batsmen for Test Matches. Journal of Quantitative Analysis in Sports. 2010;6(3):1877-2006.

16. Saikia H, Bhattacharjee D, Bhattacharjee A. Is IPL responsible for cricketers' performance in Twenty20 world cup? International Journal of Sports Science and Engineering. 2011;6(2):96-110.

17. Lemmer HH. Performance Measures for Wicket Keepers in Cricket. South African for Research in Sport, Physical Education and Recreation. 2011;33(3):89-102.

18. Knight J. Cricket for Dummies. John Wiley \& Sons, UK; 2006.

19. Hagen KV, Roach R, Summers B. The Sliding Stop: A Technique of Fielding in Cricket with a Potential for Serious Knee Injury. Br J Sports Med. 2003;34(5):379-381.

20. Camera G, Carvalho M. A tutorial on Spatial Analysis of Areas. Spatial Analysis of geographical data; 2004. p. 1-44.

21. Copas JB. The shrinkage of point scoring methods. Journal of the Royal Statistical Society. 1993;42(2):315-331.

22. Efron B, Gong G. A Leisurely Look at the Bootstrap, the Jackniffe, and Cross Validation. The American Statistician. 1983;37(1):36-48.

23. Efron B, Tibsirani R. Bootstrap Methods for Standard Errors, Confidence Intervals and other Measures of Statistical Accuracy. Statistical Science. 1986;1(1):54-75.

24. Lynch SM. Introduction to Applied Bayesian Statistics and Estimation for Social Scientist. Springer, USA; 2007. p. 335.

25. Albright S C, Winston WL, Zappe CJ. Decision Making Using Microsoft Excel. Nelson Education Ltd, Canada; 2009. p. 1-17.

26. Iyenger NS, Sudarshan P.Amethod of classifying regions from multivariate data. Economic and Political Weekly. 1982;17(51):2048-2052.

27. Duckworth FC, Lewis AJ. A fair method of for resetting the target in interrupted one-day cricket matches. Journal of the Operational Research Society. 1998;49(3):220-227.

28. Freund JE. Mathematical Statistics. Prentice Hall. USA; 1992 